$\underset{\text { Clinical }}{\text { nephron }}$

Practice
Nephron

DOI: $10.1159 / 000486323$
Received: August 28, 2017

Accepted: December 11, 2017

Published online: January 18, 2018

\title{
Association of Ultrafiltration Rate with Mortality in Incident Hemodialysis Patients
}

\author{
Tae Woo Kim ${ }^{\text {a, b }}$ Tae lk Chang ${ }^{a, c}$ Tae Hee Kim ${ }^{a, d}$ Jason A. Chou ${ }^{a}$ \\ Melissa Soohoo $^{a}$ Vanessa A. Ravel $^{\mathrm{a}}$ Csaba P. Kovesdy ${ }^{\mathrm{e}}$ \\ Kamyar Kalantar-Zadeh ${ }^{a, f}$ Elani Streja ${ }^{a, f}$
}

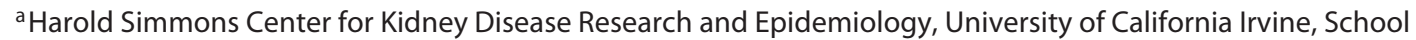
of Medicine, Orange, CA, USA; 'b Department of Internal Medicine, Soon Chun Hyang University Hospital, Gumi, South Korea; 'Department of Internal Medicine, NHIS Medical Center, Ilsan Hospital, Goyangshi, South Korea; ${ }^{\mathrm{d}}$ Department of Internal Medicine, Inje University, Busan, South Korea; ${ }^{\mathrm{e}}$ Division of Nephrology, University of

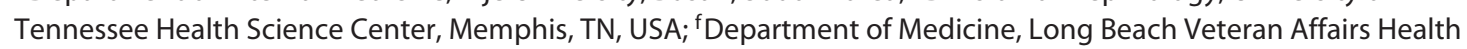
System, Long Beach, CA, USA
\end{abstract}

\section{Keywords}

Ultrafiltration · Ultrafiltration rate · Mortality $\cdot$ Hemodialysis

\begin{abstract}
Background/Aims: Ultrafiltration rate (UFR) appears to be associated with mortality in prevalent hemodialysis (HD) patients. However, the association of UFR with mortality in incident HD patients remains unknown. Methods: We examined a US cohort of 110,880 patients who initiated HD from 2007 to 2011 . Baseline UFR was divided into 5 groups $(<4,4$ to $<6,6$ to $<8,8$ to $<10$, and $\geq 10 \mathrm{~mL} / \mathrm{h} / \mathrm{kg}$ body weight [BW]). We examined predictors of higher baseline UFR using logistic regression and the association of baseline UFR and allcause and cardiovascular (CV) mortality using Cox proportional hazard models with adjustments for demographics, comorbidities, and markers of malnutrition-inflammationcachexia syndrome. Results: Patients were $63 \pm 15$ years, with $43 \%$ women, $32 \%$ African Americans, and had a mean baseline UFR of $7.5 \pm 3.1 \mathrm{~mL} / \mathrm{h} / \mathrm{kg}$ BW. In the fully adjusted logistic regression models, factors associated with higher
\end{abstract}

\section{KARGER}

(C) 2018 S. Karger AG, Basel

E-Mail karger@karger.com

www.karger.com/nef
UFR ( $\geq 7.5 \mathrm{~mL} / \mathrm{h} / \mathrm{kg} \mathrm{BW}$ ) included Hispanic ethnicity, diabetes, and higher dietary protein intake. There was a linear association between UFR and all-cause and CV mortality, where UFR $\geq 10 \mathrm{~mL} / \mathrm{h} / \mathrm{kg}$ BW (reference UFR $6-<8 \mathrm{~mL} / \mathrm{h} / \mathrm{kg}$ $\mathrm{BW}$ ) conferred the highest risk in both unadjusted (HR 1.15 [95\% Cl 1.10-1.19]) and adjusted models (HR 1.23 [95\% Cl 1.16-1.31]). The linear association with all-cause mortality remained consistent across strata of age, urine volume, and treatment time. Conclusions: Higher UFR is independently associated with higher all-cause and CV mortality in incident HD patients. Clinical trials are warranted to examine the effects of lowering UFR on outcomes. @ 2018 S. Karger AG, Basel

\section{Introduction}

Hemodialysis (HD) patients experience high mortality rates [1] and their cardiovascular (CV)-related risk of death is 10-20 times higher than that of the general population [2]. HD patients are commonly affected by prob- 
lems of volume overload or fluid retention between dialysis sessions due to lack of renal function, which can aggravate (often pre-existing) hypertension and adversely affect CV health [3]. Although previous studies have shown that higher interdialytic weight gain (IDWG) is associated with better nutritional status [4-6], it can cause volume overload and abnormal ventricular remodeling and lead to heart failure [7]. As a result of excessive IDWG, patients tend to receive a higher ultrafiltration rate (UFR) in order to remove volume during HD treatments of relatively fixed duration.

UFR is a function of the amount of fluid removed during a dialysis session (ultrafiltration) as well as the session length (dialysis treatment time). Several studies have suggested that longer dialysis treatment time is associated with better outcomes [8-11]. In addition, other studies have shown that rapid fluid removal during dialysis may contribute to all-cause and CV mortality in maintenance HD patients [12-14]. Flythe et al. [14] examined the association of UFR with mortality outcomes in 1,846 US prevalent HD patients, and reported that compared to UFR $\leq 10 \mathrm{~mL} / \mathrm{h} / \mathrm{kg}$ body weight (BW), UFR $>13 \mathrm{~mL} / \mathrm{h} / \mathrm{kg}$ $\mathrm{BW}$ was associated with higher risk of all-cause and CV mortality. However, associations in incident patients are unknown. Generally, the UFR of HD patients tends to increase as dialysis vintage increases. As a result, UFR of prevalent HD patients is more likely to be higher than that of incident HD patients.

We undertook this study in order to evaluate the association between baseline UFR and mortality in a large, contemporary cohort of incident HD patients. We hypothesized that higher UFR would be associated with higher all-cause and CV mortality.

\section{Methods}

\section{Study Population and Data}

We retrospectively examined data from all incident ESRD patients who initiated treatment between January 1,2007 and December 31,2011 , in any one of the outpatient facilities of a large dialysis organization (LDO) in the United States. The creation of our patient cohort has been previously described [15]. Patients were excluded from the cohort if treated with dialysis for less than 60 days, or treated with any modality other than in-center HD. We further excluded patients with missing UFR data in the first 91 days of $\mathrm{HD}$ and those with baseline UFR data $<0.5$ or $>18.1 \mathrm{~mL} / \mathrm{h} / \mathrm{kg} \mathrm{BW}$. The final study population consisted of 110,880 patients (online suppl. Fig. S1; for all online suppl. material, see www.karger.com/ doi/10.1159/000486323). The study was approved by the Institutional Review Board of University of California, Irvine. The requirement for a written consent was exempt due to the large sample size, patient anonymity, and non-intrusive nature of the research.

\section{Demographic, Clinical, and Laboratory Measures}

The information on self-identified race/ethnicity, primary insurance, access type, and pre-existing comorbidities were obtained from the electronic records database of the LDO. Blood samples were drawn using uniform techniques in the LDO clinics and were transported to the central laboratory in Deland, Florida, typically within $24 \mathrm{~h}$. All laboratory values were measured by automated and standardized methods. Most laboratory parameters were measured monthly, including blood urea nitrogen, serum albumin, creatinine, total-iron binding capacity, bicarbonate, phosphorous, and calcium. Ferritin was measured at least quarterly. Hemoglobin was measured at least monthly in all patients and weekly to biweekly in most patients. Kt/V was used to estimate dialysis dosage, and normalized protein catabolic rate (nPCR) was measured monthly as an indicator of daily protein intake. Most blood samples were collected before dialysis, except for post-dialysis serum urea nitrogen to calculate urea kinetics. To minimize measurement variability, all repeated clinical and laboratory measurements including UF, UFR, $24 \mathrm{~h}$ urine volume, and blood pressure for each patient during the first 91 days (quarter) of dialysis were averaged.

\section{Exposure and Outcome Variables}

Baseline ultrafiltration was measured as the weight change (post-dialysis weight minus pre-dialysis weight) during dialysis. Baseline UFR was calculated by dividing the ultrafiltration by the dialysis treatment time in hours and post-dialysis weight $[12,13]$. We used delivered UFR, and UFR was expressed in terms of $\mathrm{mL} / \mathrm{h} /$ $\mathrm{kg}$ BW. We divided UFR into 5 ordinal categories $(<4,4$ to $<6,6$ to $<8,8$ to $<10$, and $\geq 10 \mathrm{~mL} / \mathrm{h} / \mathrm{kg} \mathrm{BW}$ ) based on the distribution of the cohort. The outcomes of interest were all-cause and CV mortalities. Data on mortality were obtained from the LDO data records, and CV death was defined as death due to myocardial infarction, cardiac arrest, congestive heart failure, cerebrovascular accident, pericarditis, atherosclerotic heart disease, cardiomyopathy, cardiac arrhythmia, valvular heart disease, pulmonary edema, pulmonary embolus, or ischemic brain damage. Patients were followed up to 5 years starting from 91 days after HD initiation. Patients were censored for death, loss to follow-up, kidney transplantation, transfer to another dialysis organization, or at the end of the study period (December 31, 2011).

\section{Statistical Analyses}

Baseline characteristics were described using proportions, means $( \pm \mathrm{SD})$, or median (interquartile range), as appropriate. Data across UFR groups were compared using, chi-square, analysis of variance, and Kruskal-Wallis, as appropriate. We examined predictors of higher UFR (dichotomized at the mean UFR $\geq 7.5$ vs. $<7.5 \mathrm{mg} / \mathrm{h} / \mathrm{kg} \mathrm{BW}$ ) using logistic regression models. Finally, we examined the association of UFR with all-cause and CV mortalities using Cox proportional hazards regression models across 5 UFR categories using UFR 6 to $<8 \mathrm{~mL} / \mathrm{h} / \mathrm{kg}$ BW as reference.

We examined the relationship of baseline UFR with all-cause and CV mortality as well as predictors of UFR using 3 models of hierarchical adjustment: (i) unadjusted model that included entry calendar quarter; (ii) case-mix adjusted model that included variables in the unadjusted model plus age, sex, race/ethnicity (White, African American, Hispanic, Asian, and others), primary insurance (Medicare, Medicaid, and other), initial vascular access type (central venous catheter, arteriovenous fistula, arteriovenous graft, 
and unknown), 13 comorbid conditions (diabetes mellitus, hypertension, atherosclerotic heart disease, congestive heart failure, other CV disease, cerebrovascular disease, dyslipidemia, chronic obstructive pulmonary disease, liver disease, human immunodeficiency virus, malignancy, alcohol and substance abuse), and baseline dialysis dose as indicated by single-pool Kt/V; and (iii) case-mix and malnutrition-inflammation-cachexia syndrome (MICS) model that included all of the covariates in the case-mix model and nutritional, inflammatory surrogate markers consisting of baseline body mass index and 13 baseline laboratory variables: (1) white blood cell count, (2) lymphocyte percentage, (3) serum albumin, (4) creatinine, (5) blood urea nitrogen, (6) bicarbonate, (7) calcium, (8) phosphorus, (9) parathyroid hormone, (10) hemoglobin, (11) total-iron binding capacity, (12) ferritin, and (13) nPCR as an indicator of daily protein intake, also known as the normalized protein nitrogen appearance.

The UFR-mortality association was also examined with continuous UFR using restricted cubic splines across the 3 models of adjustment (unadjusted, case-mix, and case-mix + MICS). Best placed knots were at the 5 th, 35 th, 65 th, and 95 th percentile of the UFR distribution. We additionally examined the UFR-mortality association across a priori selected subgroups. To further examine the association of UFR-mortality, we evaluated short-term outcomes of baseline UFR with 1-year all-cause mortality.

Complete case analyses were used. Covariates used in analytical models were missing $<3 \%$ for most laboratory measurements and $6 \%$ for creatinine. Unadjusted survival models included 110,880 patients, case-mix adjusted 108,343 patients, and case-mix + MICS adjusted included 99,590 patients. In subgroup analyses, urine volume was missing in $66 \%$ of the cohort. All statistical analyses were carried out using Stata, version 13.1 (StataCorp LP, College Station, TX, USA).

\section{Results}

\section{Patient Characteristics}

The baseline demographics, clinical, and laboratory characteristics of the patients stratified by categories of baseline UFR are summarized in Table 1 . The mean age of patients was $63 \pm 15$ years, with $43 \%$ women, $32 \%$ African Americans, and 58\% diabetics. The mean baseline UFR was $7.5 \pm 3.1 \mathrm{~mL} / \mathrm{h} / \mathrm{kg}$ BW. Patients with a higher baseline UFR were younger and more likely to be Hispanic, have diabetes and congestive heart failure, lower body mass index, albumin, and lymphocyte, higher phosphorous, shorter treatment time, and a lower 24 -h urine volume, but higher ultrafiltration.

\section{Predictors of Higher UFR}

In logistic regression models adjusted for case-mix and MICS covariates, factors associated with higher UFR $(\geq 7.5 \mathrm{~mL} / \mathrm{h} / \mathrm{kg} \mathrm{BW})$ included Hispanic and Asian race (compared to White); use of arteriovenous graft as initial vascular access (compared to central venous catheter); presence of diabetes and congestive heart failure; and higher baseline nPCR, ultrafiltration, phosphorus, and blood urea nitrogen levels. However, factors associated with lower UFR $(<7.5 \mathrm{~mL} / \mathrm{h} / \mathrm{kg}$ BW) included younger age, use of arteriovenous fistula as initial vascular access (compared to central venous catheter), longer dialysis treatment time, increased baseline urine volume, and higher baseline single-pool Kt/V (Table 2). We also performed additional logistic regression analyses to examine predictors of UFR $\geq 10 \mathrm{~mL} / \mathrm{h} / \mathrm{kg} \mathrm{BW}$. After adjustment for case-mix and MICS covariates, similar factors were predictors of higher UFR $\geq 10$ as for UFR $\geq 7.5 \mathrm{~mL} / \mathrm{h} / \mathrm{kg} \mathrm{BW}$, including the presence of congestive heart failure, higher ultrafiltration, and phosphorus (online suppl. Table S1).

\section{UFR and All-Cause and CV Mortality}

During the median (interquartile range) follow-up time of $1.3(0.6-2.5)$ years, 28,896 deaths (26\%) were observed and 10,171 (35.2\%) deaths were due to CV causes. The numbers of kidney transplant, discontinuation of dialysis, and transfer to another dialysis organization were $3,762,2,201$, and 7,015, respectively. Figure 1 shows the 5 -year mortality risk across UFR groups in 110,880 patients. There was an incremental and linear association between UFR and all-cause and CV mortality across all levels of adjustment. Greatest baseline UFR $\geq 10 \mathrm{~mL} / \mathrm{h} / \mathrm{kg}$ BW was associated with higher risk of all-cause (highest risk [HR] 1.15 [95\% CI 1.10-1.19]) (reference: 6 to $<8$ $\mathrm{mL} / \mathrm{h} / \mathrm{kg} \mathrm{BW}$ ) and CV mortality (HR 1.23 (95\% CI 1.161.31) in fully adjusted models. Similar results were also observed after examining continuous UFR and all-cause and CV mortality using restricted cubic splines (Fig. 2). Moreover, in sensitivity analysis, the mortality associations were similar using baseline categorical ultrafiltration alone, and an unscaled UFR exposure (online suppl. Fig. S2, S3). Examination of short-term outcomes similarly revealed an incremental and linear association between baseline UFR and 1-year all-cause mortality across all levels of adjustment (online suppl. Fig. S4).

Figures 3 and 4 show the association of UFR and allcause mortality across various subgroups. The linear association between higher UFR and higher all-cause mortality was evident across nearly all subgroups. Notably among patients with the longest treatment time $(\geq 4.0 \mathrm{~h})$, largest UF volume $(\geq 1.7 \mathrm{~kg})$, and with the greatest urine volume $(\geq 1,100 \mathrm{~mL} /$ day $)$, the survival advantages were more prominent for patients with the lowest UFR. In addition, linear associations between UFR and mortality appear to be stronger in higher weight quintiles for both men and women. 
Table 1. Baseline characteristics of 110,880 MHD patients stratified by baseline ultrafiltration rate categories

\begin{tabular}{|c|c|c|c|c|c|c|c|}
\hline \multirow[t]{2}{*}{ Variable } & \multirow{2}{*}{$\begin{array}{l}\text { All patients } \\
(n=110,880)\end{array}$} & \multicolumn{5}{|c|}{ Ultrafiltration rate, $\mathrm{mL} / \mathrm{hr} / \mathrm{kgBW}$} & \multirow[t]{2}{*}{$p$ valu } \\
\hline & & $\begin{array}{l}<4 \\
(n=13,109)\end{array}$ & $\begin{array}{l}4 \text { to }<6 \\
(n=25,325)\end{array}$ & $\begin{array}{l}6 \text { to }<8 \\
(n=29,035)\end{array}$ & $\begin{array}{l}8 \text { to }<10 \\
(n=21,617)\end{array}$ & $\begin{array}{l}\geq 10 \\
(n=21,794)\end{array}$ & \\
\hline Age, years & $63 \pm 15$ & $63 \pm 15$ & $63 \pm 14$ & $63 \pm 15$ & $63 \pm 15$ & $61 \pm 16$ & $<0.001$ \\
\hline Female, \% & 43 & 49 & 46 & 42 & 39 & 42 & $<0.001$ \\
\hline \multicolumn{8}{|l|}{ Race, $\%$} \\
\hline White & 47 & 54 & 49 & 46 & 45 & 43 & $<0.001$ \\
\hline African American & 32 & 30 & 34 & 33 & 31 & 28 & $<0.001$ \\
\hline Hispanic & 15 & 10 & 12 & 14 & 17 & 19 & $<0.001$ \\
\hline Asian & 3 & 2 & 2 & 3 & 4 & 5 & $<0.001$ \\
\hline Others & 3 & 4 & 3 & 4 & 3 & 5 & $<0.001$ \\
\hline \multicolumn{8}{|l|}{ Primary insurance, $\%$} \\
\hline Medicare & 54 & 53 & 53 & 54 & 54 & 53 & 0.022 \\
\hline Medicaid & 7 & 5 & 6 & 6 & 7 & 10 & $<0.001$ \\
\hline Others & 39 & 42 & 41 & 40 & 39 & 37 & $<0.001$ \\
\hline \multicolumn{8}{|l|}{ Access, \% } \\
\hline CVC & 74 & 72 & 73 & 74 & 74 & 76 & $<0.001$ \\
\hline $\mathrm{AVF}$ & 15 & 17 & 16 & 15 & 15 & 13 & $<0.001$ \\
\hline AVG & 4 & 4 & 4 & 4 & 4 & 4 & 0.049 \\
\hline Unknown & 7 & 7 & 7 & 7 & 7 & 7 & $<0.001$ \\
\hline \multicolumn{8}{|l|}{ Comorbidity, \% } \\
\hline Diabetes & 58 & 52 & 58 & 60 & 59 & 59 & $<0.001$ \\
\hline Hypertension & 51 & 52 & 51 & 51 & 52 & 50 & 0.011 \\
\hline Congestive heart failure & 36 & 28 & 34 & 37 & 39 & 42 & $<0.001$ \\
\hline Atherosclerotic heart disease & 14 & 14 & 14 & 15 & 14 & 14 & 0.152 \\
\hline Other cardiovascular disease & 15 & 15 & 15 & 15 & 15 & 15 & 0.163 \\
\hline Dyslipidemia & 25 & 26 & 26 & 25 & 25 & 24 & $<0.001$ \\
\hline Chronic obstructive pulmonary disease & 5 & 5 & 5 & 5 & 5 & 5 & 0.009 \\
\hline Liver disease & 1 & 1 & 1 & 1 & 2 & 2 & 0.08 \\
\hline Cerebrovascular disease & 2 & 2 & 2 & 2 & 2 & 2 & 0.027 \\
\hline History of cancer & 2 & 3 & 3 & 2 & 2 & 2 & $<0.001$ \\
\hline Human immunodeficiency virus & 0.5 & 0.5 & 0.4 & 0.4 & 0.5 & 0.6 & 0.005 \\
\hline Substance abuse & 0.2 & 0.2 & 0.2 & 0.2 & 0.3 & 0.4 & $<0.001$ \\
\hline Alcohol abuse & 0.2 & 0.2 & 0.2 & 0.2 & 0.3 & 0.4 & 0.001 \\
\hline Body mass index, $\mathrm{kg} / \mathrm{m}^{2}$ & $28.2 \pm 7.3$ & $30.6 \pm 8.5$ & $30.6 \pm 8.2$ & $28.8 \pm 7.1$ & $26.8 \pm 5.9$ & $24.4 \pm 4.9$ & $<0.001$ \\
\hline Single-pool, Kt/V & $1.47 \pm 0.32$ & $1.48 \pm 0.34$ & $1.45 \pm 0.32$ & $1.46 \pm 0.32$ & $1.46 \pm 0.32$ & $1.49 \pm 0.32$ & $<0.001$ \\
\hline nPCR, g/kg/day & $0.8 \pm 0.2$ & $0.7 \pm 0.2$ & $0.8 \pm 0.2$ & $0.8 \pm 0.2$ & $0.8 \pm 0.2$ & $0.8 \pm 0.2$ & $<0.001$ \\
\hline Weekday IDWG, \% & $2.3(1.5-3.1)$ & $0.9(0.5-1.2)$ & $1.6(1.3-1.9)$ & $2.2(1.9-2.6)$ & $2.8(2.4-3.2)$ & $3.7(3.2-4.4)$ & $<0.001$ \\
\hline Weekend IDWG, \% & $3.1(2.1-4.2)$ & $1.3(0.8-1.7)$ & $2.2(1.7-2.7)$ & $3.0(2.5-3.5)$ & $3.8(3.2-4.4)$ & $5.0(4.2-5.9)$ & $<0.001$ \\
\hline Ultrafiltration, $\mathrm{kg}$ & $2.0 \pm 0.9$ & $0.9 \pm 0.4$ & $1.6 \pm 0.6$ & $2.1 \pm 0.6$ & $2.4 \pm 0.7$ & $2.8 \pm 0.8$ & $<0.001$ \\
\hline Dialysis treatment time, $\mathrm{min} /$ session & $211.5 \pm 23.5$ & $214.4 \pm 25.2$ & $215.6 \pm 23.6$ & $213.2 \pm 22.7$ & $210.1 \pm 22.5$ & $204.0 \pm 22.3$ & $<0.001$ \\
\hline $24-\mathrm{H}$ urine volume, $\mathrm{mL} /$ day & $775(450-1,300)$ & $1,000(550-1,525)$ & $825(500-1,350)$ & $750(408-1,250)$ & $700(400-1,200)$ & $625(350-1,100)$ & $<0.001$ \\
\hline Pre-dialysis SBP, mm Hg & $147.0 \pm 19.4$ & $144.4 \pm 19.0$ & $146.2 \pm 18.8$ & $147.5 \pm 19.6$ & $147.5 \pm 19.6$ & $148.9 \pm 20.3$ & $<0.001$ \\
\hline Post-dialysis SBP, mm Hg & $144.1 \pm 18.3$ & $143.9 \pm 18.6$ & $144.2 \pm 18.0$ & $144.0 \pm 18.0$ & $144.0 \pm 18.3$ & $144.3 \pm 19.1$ & 0.122 \\
\hline Lowest intradialytic SBP, $\mathrm{mm} \mathrm{Hg}$ & $116.0 \pm 15.1$ & $115.4 \pm 15.7$ & $116.0 \pm 15.1$ & $116.3 \pm 15.2$ & $116.7 \pm 15.6$ & $117.7 \pm 16.3$ & $<0.001$ \\
\hline $\begin{array}{c}\text { Frequency of Lowest intradialytic } \\
\text { hypotension, }<90 \mathrm{~mm} \mathrm{Hg}\end{array}$ & $1(0-4)$ & $1(0-4)$ & $1(0-4)$ & $1(0-4)$ & $1(0-4)$ & $1(0-4)$ & $<0.001$ \\
\hline \multicolumn{8}{|l|}{ Laboratory parameters } \\
\hline Hemoglobin, g/dL & $11.1 \pm 1.2$ & $11.2 \pm 1.2$ & $11.1 \pm 1.2$ & $11.1 \pm 1.2$ & $11.1 \pm 1.2$ & $11.0 \pm 1.2$ & $<0.001$ \\
\hline White blood cells, $\times 10^{3} / \mu \mathrm{L}$ & $7.8 \pm 2.7$ & $7.9 \pm 2.7$ & $7.9 \pm 2.6$ & $7.8 \pm 2.7$ & $7.7 \pm 2.7$ & $7.8 \pm 2.8$ & $<0.001$ \\
\hline Lymphocyte (\% of white blood cells) & $20.7 \pm 7.5$ & $21.7 \pm 7.6$ & $21.3 \pm 7.5$ & $20.8 \pm 7.5$ & $20.4 \pm 7.5$ & $19.6 \pm 7.4$ & $<0.001$ \\
\hline Albumin, $\mathrm{g} / \mathrm{dL}$ & $3.5 \pm 0.5$ & $3.6 \pm 0.5$ & $3.6 \pm 0.5$ & $3.5 \pm 0.5$ & $3.5 \pm 0.5$ & $3.4 \pm 0.5$ & $<0.001$ \\
\hline Calcium, mg/dL & $9.1 \pm 0.6$ & $9.2 \pm 0.6$ & $9.1 \pm 0.6$ & $9.1 \pm 0.6$ & $9.1 \pm 0.6$ & $9.0 \pm 0.6$ & $<0.001$ \\
\hline Phosphorus, mg/dL & $4.9 \pm 1.1$ & $4.7 \pm 1.0$ & $4.8 \pm 1.1$ & $4.9 \pm 1.1$ & $5.0 \pm 1.2$ & $5.2 \pm 1.3$ & $<0.001$ \\
\hline Blood urea nitrogen, $\mathrm{mg} / \mathrm{dL}$ & $48 \pm 15$ & $44 \pm 14$ & $46 \pm 14$ & $48 \pm 14$ & $50 \pm 15$ & $52 \pm 15$ & $<0.001$ \\
\hline Creatinine, $\mathrm{mg} / \mathrm{dL}$ & $5.9 \pm 2.4$ & $5.8 \pm 2.2$ & $5.9 \pm 2.3$ & $5.9 \pm 2.3$ & $5.9 \pm 2.4$ & $5.9 \pm 2.5$ & $<0.001$ \\
\hline Bicarbonate, $\mathrm{mEq} / \mathrm{L}$ & $23.6 \pm 2.7$ & $23.5 \pm 2.8$ & $23.7 \pm 2.7$ & $23.7 \pm 2.7$ & $23.6 \pm 2.7$ & $23.4 \pm 2.7$ & $<0.001$ \\
\hline Intact parathyroid hormone, $\mathrm{pg} / \mathrm{mL}$ & $314(197-486)$ & $313(191-498)$ & $320(201-499)$ & $318(201-489)$ & $312(198-482)$ & $302(190-467)$ & $<0.001$ \\
\hline Ferritin, pg/nL & $282(164-485)$ & $285(165-489)$ & $280(164-481)$ & $279(163-476)$ & $281(163-485)$ & $291(166-500)$ & $<0.001$ \\
\hline Total iron biding capacity, mg/dL & $224.8 \pm 49.0$ & $228.3 \pm 51.1$ & $226.3 \pm 49.3$ & $225.3 \pm 48.2$ & $223.8 \pm 48.6$ & $221.3 \pm 48.7$ & $<0.001$ \\
\hline
\end{tabular}

Data are presented as mean $\pm \mathrm{SD}$, median (IQR), or percentage, appropriately.

nPCR, normalized protein catabolic rate; SBP, systolic blood pressure; MHD, maintenance hemodialysis; IDWG, interdialytic weight gain. 
Table 2. Multivariate logistic regression model predicting UFR $\geq 7.5 \mathrm{~mL} / \mathrm{h} / \mathrm{kg}$ BW in 110,880 MHD patients

\begin{tabular}{|c|c|c|c|c|c|c|}
\hline \multirow[t]{2}{*}{ Variable } & \multicolumn{2}{|l|}{ Unadjusted } & \multicolumn{2}{|l|}{ Case-mix } & \multicolumn{2}{|l|}{ Case-mix + MICS } \\
\hline & OR (95\% CI) & $p$ value & OR (95\% CI) & $p$ value & OR $(95 \% \mathrm{CI})$ & $p$ value \\
\hline Age $(\Delta 10$ years $)$ & $0.95(0.94-0.96)$ & $<0.001$ & $0.96(0.95-0.97)$ & $<0.001$ & $0.88(0.87-0.89)$ & $<0.001$ \\
\hline Female & $0.83(0.81-0.85)$ & $<0.001$ & $0.79(0.77-0.81)$ & $<0.001$ & $1.11(1.08-1.15)$ & $<0.001$ \\
\hline \multicolumn{7}{|l|}{ Race } \\
\hline White & Reference & & Reference & & Reference & \\
\hline African American & $1.01(0.98-1.04)$ & 0.607 & $0.99(0.96-1.02)$ & 0.543 & $1.17(1.13-1.21)$ & $<0.001$ \\
\hline Hispanic & $1.56(1.51-1.62)$ & $<0.001$ & $1.49(1.43-1.54)$ & $<0.001$ & $1.30(1.25-1.36)$ & $<0.001$ \\
\hline Asian & $2.12(1.98-2.27)$ & $<0.001$ & $2.08(1.94-2.23)$ & $<0.001$ & $1.36(1.25-1.47)$ & $<0.001$ \\
\hline Others & $1.33(1.24-1.41)$ & $<0.001$ & $1.29(1.21-1.38)$ & $<0.001$ & $1.18(1.25-1.36)$ & $<0.001$ \\
\hline \multicolumn{7}{|l|}{ Primary insurance } \\
\hline Medicare & Reference & & Reference & & Reference & \\
\hline Medicaid & $1.41(1.34-1.48)$ & $<0.001$ & $1.21(1.15-1.28)$ & $<0.001$ & $1.12(1.06-1.19)$ & $<0.001$ \\
\hline Others & $0.93(0.91-0.96)$ & $<0.001$ & $0.89(0.86-0.91)$ & $<0.001$ & $0.90(0.87-0.92)$ & $<0.001$ \\
\hline \multicolumn{7}{|l|}{ Access } \\
\hline CVC & Reference & & Reference & & Reference & \\
\hline AVF & $0.84(0.81-0.87)$ & $<0.001$ & $0.85(0.82-0.88)$ & $<0.001$ & $0.93(0.90-0.97)$ & $<0.001$ \\
\hline AVG & $1.03(0.97-1.10)$ & 0.282 & $1.10(1.03-1.17)$ & 0.003 & $1.30(1.22-1.40)$ & $<0.001$ \\
\hline Unknown & $0.90(0.61-1.32)$ & 0.578 & $0.94(0.63-1.39)$ & 0.756 & $1.03(0.65-1.64)$ & 0.891 \\
\hline \multicolumn{7}{|l|}{ Comorbidity } \\
\hline Diabetes & $1.07(1.05-1.10)$ & $<0.001$ & $1.03(1.00-1.06)$ & 0.02 & $1.11(1.08-1.15)$ & $<0.001$ \\
\hline Hypertension & $0.97(0.95-1.00)$ & 0.023 & $0.99(0.96-1.01)$ & 0.383 & $0.99(0.97-1.02)$ & 0.718 \\
\hline Congestive heart failure & $1.30(1.27-1.33)$ & $<0.001$ & $1.31(1.28-1.35)$ & $<0.001$ & $1.43(1.39-1.47)$ & $<0.001$ \\
\hline Atherosclerotic heart disease & $1.01(0.97-1.04)$ & 0.648 & $0.98(0.94-1.02)$ & 0.314 & $0.99(0.95-1.04)$ & 0.738 \\
\hline Other cardiovascular disease & $1.04(1.01-1.08)$ & 0.014 & $1.07(1.03-1.11)$ & 0.001 & $1.05(1.00-1.10)$ & 0.032 \\
\hline Dyslipidemia & $0.94(0.91-0.96)$ & $<0.001$ & $0.91(0.89-0.94)$ & $<0.001$ & $0.93(0.90-0.96)$ & $<0.001$ \\
\hline Chronic obstructive pulmonary disease & $0.98(0.93-1.03)$ & 0.423 & $0.96(0.91-1.02)$ & 0.24 & $1.04(0.98-1.12)$ & 0.204 \\
\hline Liver disease & $1.15(1.04-1.27)$ & 0.005 & $1.09(0.98-1.21)$ & 0.126 & $0.94(0.83-1.06)$ & 0.287 \\
\hline Cerebrovascular disease & $0.88(0.90-0.96)$ & 0.006 & $0.85(0.77-0.94)$ & 0.001 & $0.81(0.73-0.91)$ & $<0.001$ \\
\hline History of cancer & $0.72(0.67-0.79)$ & $<0.001$ & $0.75(0.69-0.81)$ & $<0.001$ & $0.70(0.63-0.76)$ & $<0.001$ \\
\hline Human immunodeficiency virus & $1.31(1.10-1.55)$ & 0.002 & $1.23(1.03-1.46)$ & 0.021 & $0.86(0.70-1.04)$ & 0.121 \\
\hline Substance abuse & $1.55(1.22-1.79)$ & $<0.001$ & $1.37(1.06-1.76)$ & 0.014 & $1.09(0.82-1.45)$ & 0.551 \\
\hline Alcohol abuse & $1.67(1.30-2.13)$ & $<0.001$ & $1.50(1.16-1.93)$ & 0.002 & $1.35(1.02-1.79)$ & 0.037 \\
\hline Body mass index, $\mathrm{kg} / \mathrm{m}^{2}$ & $0.91(0.91-0.92)$ & $<0.001$ & $0.90(0.90-0.90)$ & $<0.001$ & $0.89(0.89-0.90)$ & $<0.001$ \\
\hline Single-pool Kt/V $(\Delta 0.1)$ & $1.01(1.01-1.01)$ & $<0.001$ & $1.02(1.02-1.02)$ & $<0.001$ & $0.94(0.93-0.94)$ & $<0.001$ \\
\hline $\operatorname{nPCR}(\Delta 0.1 \mathrm{~g} / \mathrm{kg} /$ day $)$ & $1.14(1.14-1.15)$ & $<0.001$ & $1.14(1.13-1.15)$ & $<0.001$ & $1.20(1.18-1.21)$ & $<0.001$ \\
\hline Weekday IDWG $(\Delta 0.1 \%)$ & $1.12(1.12-1.12)$ & $<0.001$ & $1.09(1.09-1.09)$ & $<0.001$ & $1.10(1.09-1.10)$ & $<0.001$ \\
\hline Weekend IDWG $(\Delta 0.1 \%)$ & $1.09(1.09-1.09)$ & $<0.001$ & $1.06(1.06-1.06)$ & $<0.001$ & $1.06(1.06-1.06)$ & $<0.001$ \\
\hline Ultrafiltration $(\Delta 0.1 \mathrm{~kg})$ & $1.20(1.20-1.21)$ & $<0.001$ & $1.26(1.25-1.26)$ & $<0.001$ & $1.67(1.66-1.68)$ & $<0.001$ \\
\hline Dialysis treatment time $(\Delta 10 \mathrm{~min} / \mathrm{session})$ & $0.88(0.88-0.89)$ & $<0.001$ & $0.86(0.85-0.86)$ & $<0.001$ & $0.92(0.91-0.92)$ & $<0.001$ \\
\hline $24-\mathrm{H}$ urine volume $(\Delta 100 \mathrm{~mL} /$ day $)$ & $0.96(0.96-0.97)$ & $<0.001$ & $0.95(0.94-0.95)$ & $<0.001$ & $0.95(0.95-0.96)$ & $<0.001$ \\
\hline Pre-dialysis SBP $(\Delta 10 \mathrm{~mm} \mathrm{Hg})$ & $1.05(1.04-1.06)$ & $<0.001$ & $1.05(1.04-1.06)$ & $<0.001$ & $1.06(1.05-1.06)$ & $<0.001$ \\
\hline Post-dialysis SBP $(\Delta 10 \mathrm{~mm} \mathrm{Hg})$ & $1.00(0.99-1.00)$ & 0.36 & $0.99(0.99-1.00)$ & 0.042 & $0.96(096-0.97)$ & $<0.001$ \\
\hline Lowest intradialytic SBP $(\Delta 10 \mathrm{~mm} \mathrm{Hg})$ & $1.04(1.03-1.05)$ & $<0.001$ & $1.04(1.03-1.05)$ & $<0.001$ & $1.0(1.01-1.02)$ & $<0.001$ \\
\hline Frequency of intradialytic hypotension $(<90 \mathrm{~mm} \mathrm{Hg})$ events (per 5 units) & $1.00(0.98-1.01)$ & 0.505 & $1.01(0.99-1.02)$ & 0.225 & $1.02(1.00-1.03)$ & 0.037 \\
\hline \multicolumn{7}{|l|}{ Laboratory parameters } \\
\hline Hemoglobin, g/dL & $0.94(0.94-0.95)$ & $<0.001$ & $0.93(0.92-0.94)$ & $<0.001$ & $0.91(0.90-0.92)$ & $<0.001$ \\
\hline White blood cells, $\times 10^{3} / \mu \mathrm{L}$ & $0.99(0.98-0.99)$ & $<0.001$ & $0.99(0.98-0.99)$ & $<0.001$ & $0.98(0.97-0.98)$ & $<0.001$ \\
\hline Lymphocyte (\% of white blood cells) & $0.98(0.98-0.98)$ & $<0.001$ & $0.98(0.98-0.98)$ & $<0.001$ & $0.98(0.98-0.98)$ & $<0.001$ \\
\hline Albumin $(\Delta 0.1 \mathrm{~g} / \mathrm{dL})$ & $0.95(0.95-0.96)$ & $<0.001$ & $0.95(0.95-0.95)$ & $<0.001$ & $0.9490 .94-0.94)$ & $<0.001$ \\
\hline Calcium, mg/dL & $0.72(0.71-0.74)$ & $<0.001$ & $0.76(0.75-0.78)$ & $<0.001$ & $0.73(0.71-0.75)$ & $<0.001$ \\
\hline Phosphorus, mg/dL & $1.22(1.21-1.24)$ & $<0.001$ & $1.22(1.20-1.23)$ & $<0.001$ & $1.24(1.22-1.26)$ & $<0.001$ \\
\hline Blood urea nitrogen $(\Delta 10 \mathrm{mg} / \mathrm{dL})$ & $1.21(1.20-1.22)$ & $<0.001$ & $1.19(1.18-1.20)$ & $<0.001$ & $1.03(1.01-1.05)$ & $<0.001$ \\
\hline Creatinine, $\mathrm{mg} / \mathrm{dL}$ & $1.00(1.00-1.01)$ & 0.105 & $0.98(0.98-0.99)$ & $<0.001$ & $0.91(0.91-0.92)$ & $<0.001$ \\
\hline Bicarbonate, $\mathrm{mEq} / \mathrm{L}$ & $0.98(0.98-0.98)$ & $<0.001$ & $0.99(0.98-0.99)$ & $<0.001$ & $1.04(1.03-1.04)$ & $<0.001$ \\
\hline Intact parathyroid hormone $(\Delta 100 \mathrm{pg} / \mathrm{mL})$ & $0.99(0.98-0.99)$ & $<0.001$ & $0.98(0.98-0.99)$ & $<0.001$ & $0.98(0.97-0.98)$ & $<0.001$ \\
\hline Ferritin $(\Delta 100 \mathrm{pg} / \mathrm{nL})$ & $1.01(1.00-1.01)$ & $<0.001$ & $1.01(1.00-1.01)$ & $<0.001$ & $0.99(0.99-0.99)$ & $<0.001$ \\
\hline Total iron biding capacity $(\Delta 100 \mathrm{mg} / \mathrm{dL})$ & $0.86(0.84-0.88)$ & $<0.001$ & $0.84(0.82-0.86)$ & $<0.001$ & $1.22(1.18-1.27)$ & $<0.001$ \\
\hline
\end{tabular}

Adjustments in unadjusted models: mortality data in each calendar quarter; case-mix adjusted models: unadjusted model plus baseline characteristics of age, sex, race/ethnicity (White, African American, Hispanic, Asian, or other), primary insurance (Medicare, Medicaid, and others), vascular access type (central venous catheter, arteriovenous fistula, arteriovenous graft, or others), 13 comorbid conditions (diabetes mellitus, hypertension, atherosclerotic heart disease, congestive heart failure, other-cardiovascular disease, cerebrovascular disease, dyslipidemia, HIV, chronic obstructive pulmonary disease, liver disease and malignancy, alcohol and substance abuse), and dialysis dose as indicated by single-pool Kt/V; case-mix plus malnutrition-inflammation-cachexia syndrome (MICS) models: case-mix adjusted model plus BMI and 13 laboratory variables including white blood cell count, lymphocyte percentage, serum albumin, creatinine, blood urea nitrogen, bicarbonate, calcium, phosphorus, parathyroid hormone, hemoglobin, total iron binding capacity, ferritin, and nPCR. nPCR, normalized protein catabolic rate; SBP, systolic blood pressure; IDWG, interdialytic weight gain; MHD, maintenance hemodialysis. 


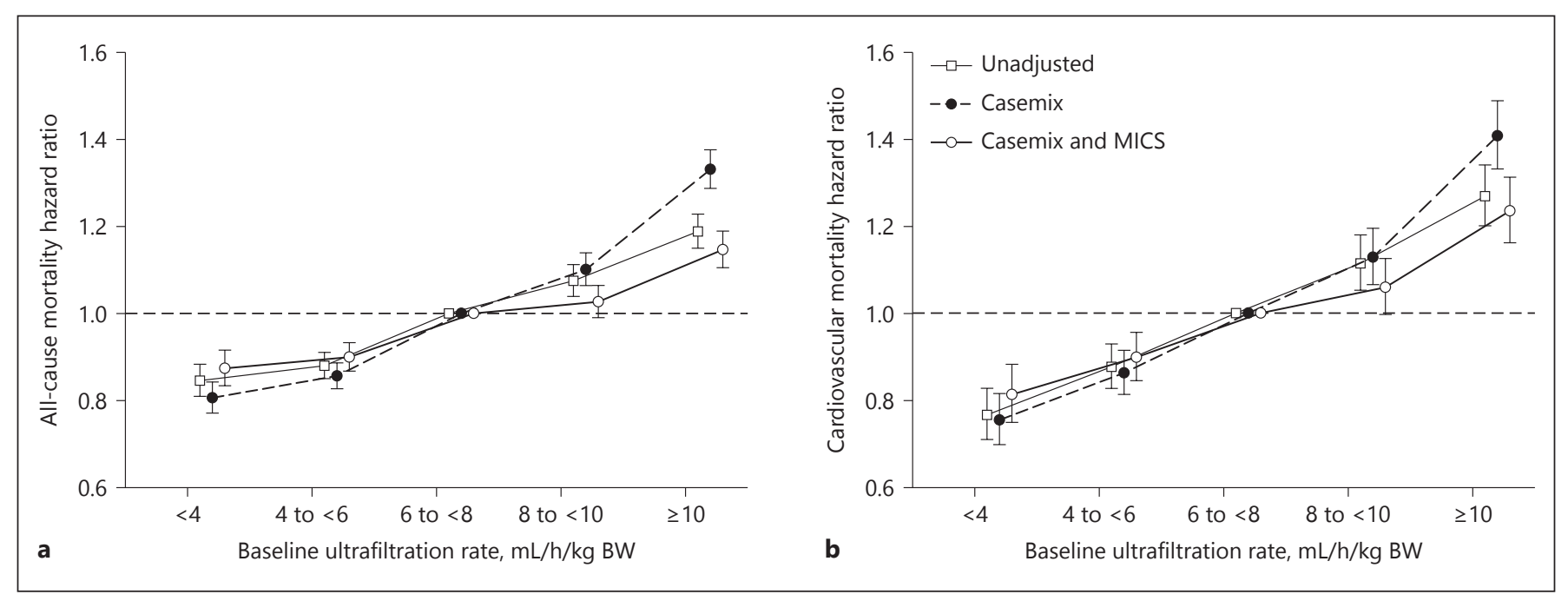

Fig. 1. Association between baseline UFR with (a) all-cause and (b) cardiovascular mortality in 110,880 incident hemodialysis patients.

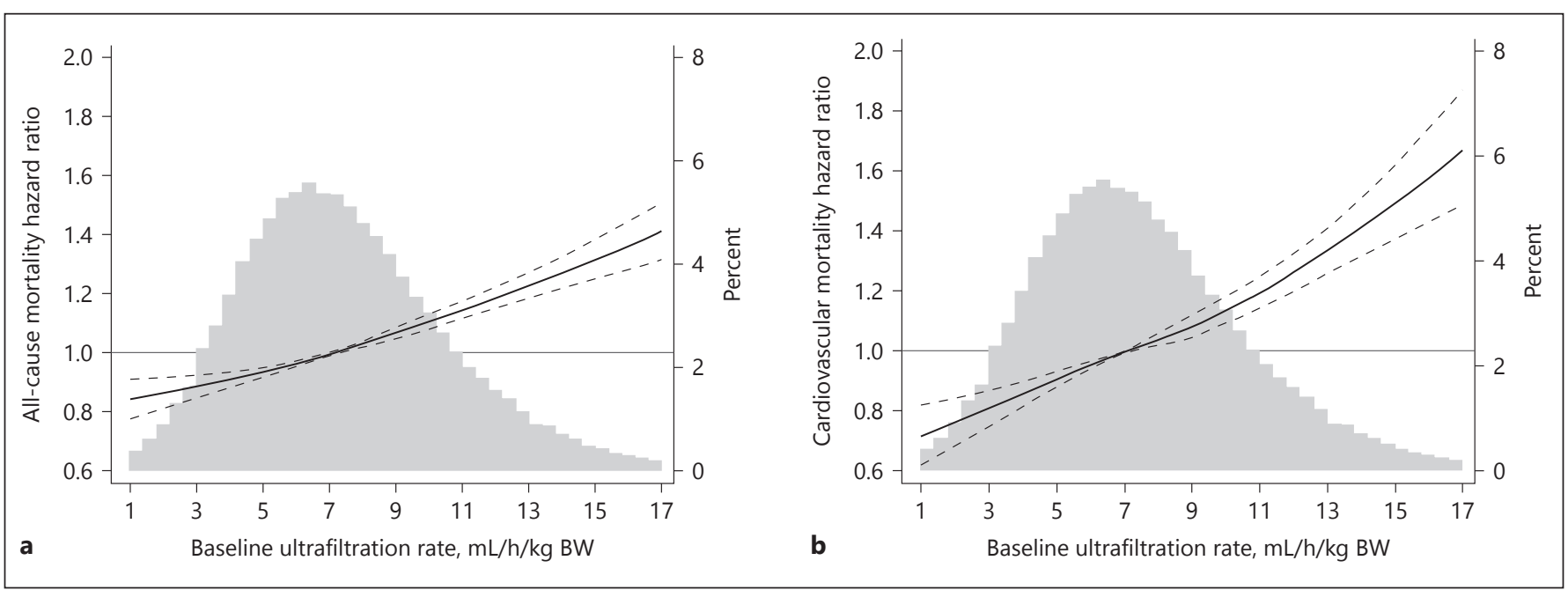

Fig. 2. Restricted cubic spine models of the association of UFR with (a) all-cause and (b) cardiovascular mortality in 110,880 incident hemodialysis patients in the case-mix and MICS-adjusted model.

\section{Discussion}

In a nationally representative cohort of 110,880 incident HD patients, UFR exhibited a direct linear relationship with higher all-cause and $\mathrm{CV}$ mortality. Factors associated with higher UFR $(\geq 7.5 \mathrm{~mL} / \mathrm{h} / \mathrm{kg} \mathrm{BW})$ included Hispanic and Asian race; use of arteriovenous graft as initial vascular access; presence of diabetes and congestive heart failure; and higher baseline nPCR, ultrafiltration, phosphorus, and blood urea nitrogen levels.
Few studies have previously examined the association between UFR with all-cause and CV mortality [12-14]. In the international DOPPS cohort, including 22,000 prevalent HD patients, Saran et al. [12] reported a significant difference in all-cause mortality risk between patients with $\mathrm{UFR} \leq 10$ versus $>10 \mathrm{~mL} / \mathrm{h} / \mathrm{kg}$ BW (relative risk [RR] $1.09, p=0.02)$; however, this difference was not evident when examining CV mortality ( RR 1.04, $p=0.41$ ). In addition, in a cohort of 287 Italian MHD prevalent patients with a mean UFR of $12.7 \pm 3.5 \mathrm{~mL} / \mathrm{h} / \mathrm{kg} \mathrm{BW}$, Movilli et al. [13] reported that better survival was observed with UFR 
Fig. 3. Association of baseline UFR with all-cause mortality a priori select subgroups of incident hemodialysis patients in the case-mix and MICS adjusted model. HD time, hemodialysis treatment time (h); $24 \mathrm{UV}, 24$-h urine volume (mL/day); post SBP, post-dialysis systolic blood pressure (mm Hg); SPKTV, single-pool Kt/V.

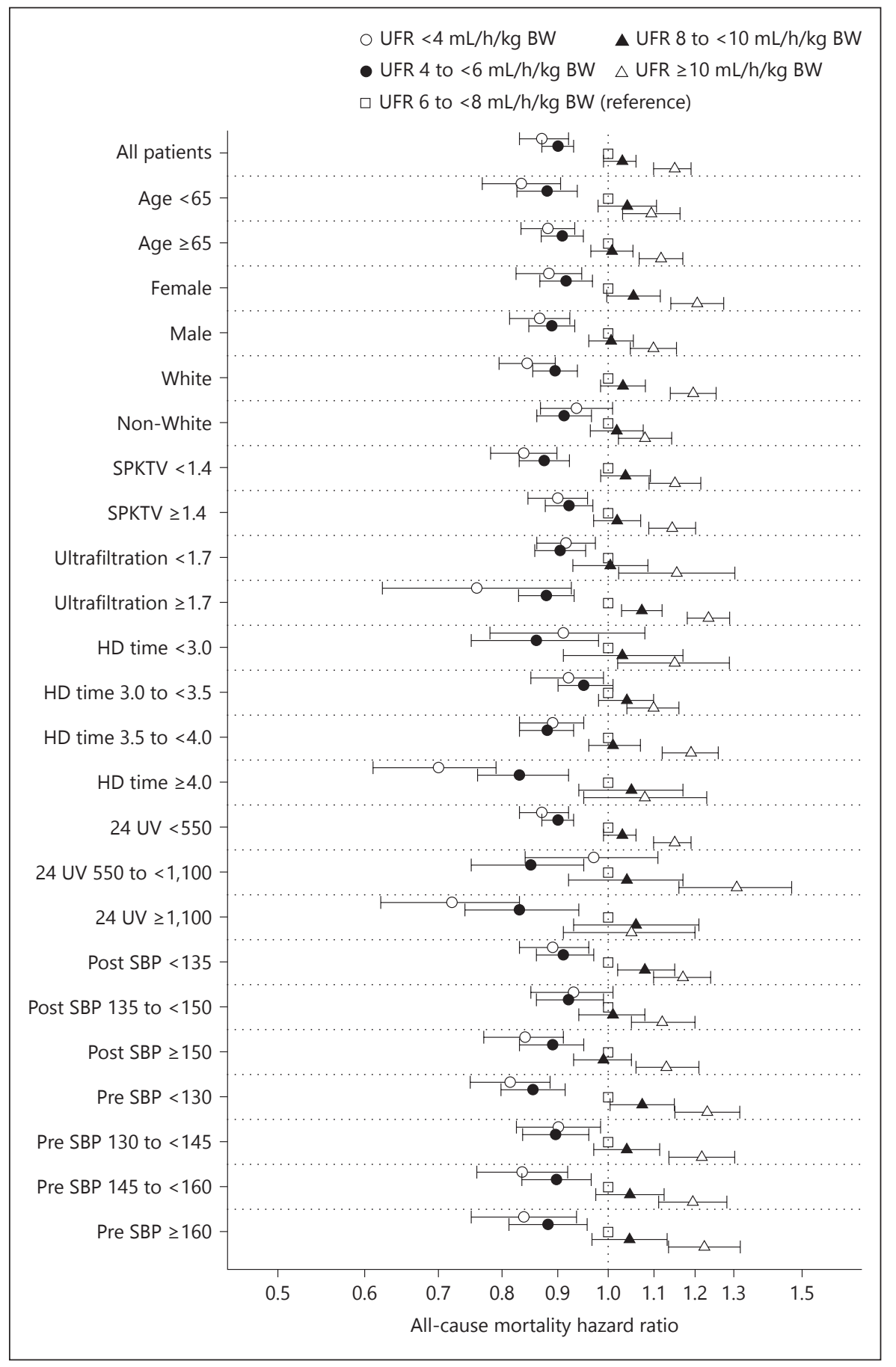

$<12.37 \mathrm{~mL} / \mathrm{h} / \mathrm{kg} \mathrm{BW}(p<0.0001)$ by evaluating receiver operator curve-generated thresholds for mortality. They also commented that a threshold of $10 \mathrm{~mL} / \mathrm{h} / \mathrm{kg}$ BW might be too low to demonstrate a true association between UFR and CV mortality as reported by Saran et al. [12] Lastly, Flythe et al. [14] reported that UFR $>13 \mathrm{~mL} / \mathrm{h} /$ $\mathrm{kg} \mathrm{BW}$ (compared to UFR $\leq 10 \mathrm{~mL} / \mathrm{h} / \mathrm{kg} \mathrm{BW}$ ) was signif- icantly associated with both all-cause and CV mortality in 1,846 prevalent US patients. Compared to the aforementioned studies, baseline UFR of our cohort was lower (mean UFR: $7.5 \pm 3.1 \mathrm{~mL} / \mathrm{h} / \mathrm{kg} \mathrm{BW}$ ). This difference is likely due to the fact that our cohort was comprised of incident patients, whereas previous studies had investigated cohorts of prevalent patients. Generally, in HD pa- 
Fig. 4. Association of baseline UFR with all-cause mortality across subgroups of sex adjusted quintiles of post-dialysis weight (kg) among incident hemodialysis patients in the case-mix and MICS-adjusted model. $\mathrm{F}$, female; $\mathrm{M}$, male; quint, quintile.

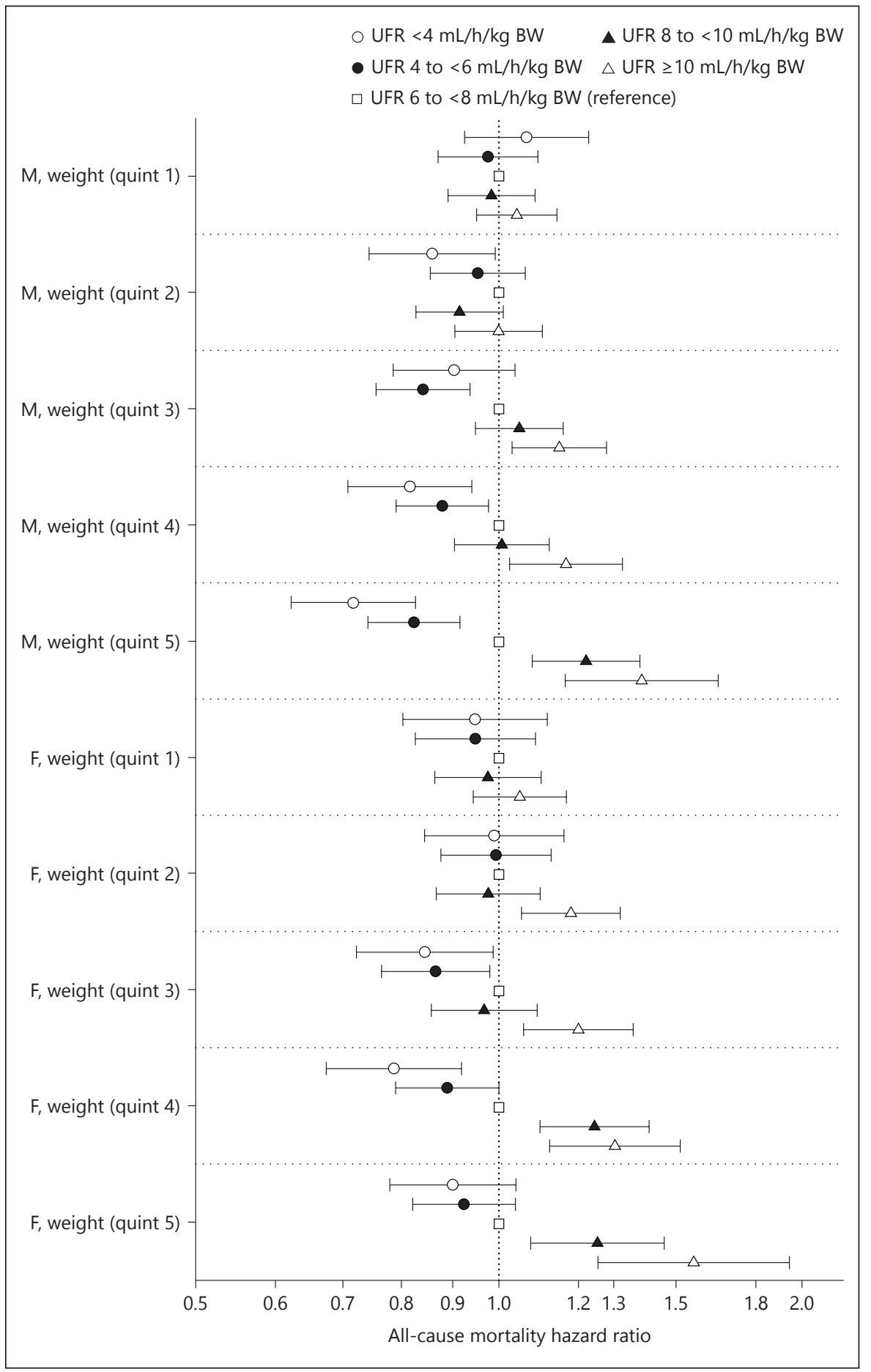

tients, over time on maintenance $\mathrm{HD}$, residual renal function decreases whereas patient appetite improves and nutritional intake increases [3-6], resulting in more sodium and water intake between dialysis sessions. As a consequence, more fluid retention can occur between dialysis sessions as patients undergo maintenance HD for longer periods of time; thereby resulting in larger ultrafiltration volumes and higher UFRs in prevalent patients to remove excess fluid.

Clinically, ultrafiltration is equivalent to IDWG at steady state. Converse to our findings, Lopez-Gomez et al. [16] reported that greater IDWG was associated with bet- 
ter long-term prognosis of patients. They found that the positive effect of higher IDWG (or fluid retention) on appetite and nutrition outweighs its negative effect on blood pressure. However, several studies have showed opposite results regarding associations between higher IDWG and mortality $[17,18]$. In a study on non-adherence in HD patients using the international prospective DOPPS cohort, Saran et al. [19] reported that IDWG over 5.7\% of BW was associated with higher mortality (RR 1.12, $p=$ 0.047 ) over a median of $1.8-3$ years of follow-up. In addition, Kalantar-Zadeh et al. [20] verified that greater fluid retention between 2 subsequent $\mathrm{HD}$ treatment sessions was associated with higher risk of all-cause and CV death in a 2-year cohort of 34,107 MHD US patients. Similarly, in our study, as ultrafiltration increased, hazards ratios of all-cause mortality risk proportionally increased in casemix and MICS-adjusted models (online suppl. Fig. S2), as well as in examination of short-term outcomes.

UFR is the ratio of fluid removed to dialysis treatment time. By increasing dialysis treatment time, HD patients become more tolerant of ultrafiltration, increase removal of uremic toxins, have less frequent intradialytic hypotensive episodes, and gain better control of blood pressure. As a result, these processes may improve $\mathrm{CV}$ and all-cause mortality outcomes. Intradialytic hypotension can cause not only dialysis intolerance but also repeated myocardial ischemia and infarction [21] and these may contribute to the development of heart failure and higher mortality risk in MHD patients [22, 23]. Recently, several investigators reconfirmed the importance of treatment time in MHD patients. Miller et al. [24] examined associations of delivered HD dose and treatment time with survival in a cohort of 88,153 US HD patients. Patients receiving a reported thriceweekly treatment time $<3 \mathrm{~h}$ per HD session (vs. $\geq 3.5 \mathrm{~h}$ ) was associated with higher death risk independent of HD dose. Flythe et al. [25] sought to further understand the relationship of the components of UFR (namely IDWG and treatment time) by examining the association each factor with mortality adjusted for the other factor, respectively. In 14,643 prevalent US MHD patients with a median follow up time of 25 months, they reported that shorter dialysis session length (<240 $\mathrm{min})$ was associated with higher allcause mortality (HR 1.32 [95\% CI 1.03-1.69]) in models adjusted for IDWG. Similar to the aforementioned studies, we too observed that patients with the longest dialysis treatment time had better survival, especially among the lower UFR groups. In addition, lower mortality risk was also observed among patients with the largest ultrafiltration $(\geq 1.7$ $\mathrm{kg}$ ) and lowest UFR group. Though this group was a small proportion of our total cohort $(<1 \%)$, they also had a rela- tively long treatment time with a median of $4.0 \mathrm{~h}$ per session, further adding to the notion that long treatment time may improve patient outcomes. Twardowski [26] stressed the importance of treatment time and UFR rather than small molecular clearance $\left(\mathrm{Kt} / \mathrm{V}_{\text {urea }}\right)$ in assessing dialysis quality. In our study, associations between UFR and mortality were similar across strata of single-pool Kt/V.

In addition, in our cohort, there was significant effect modification on the association between UFR and mortality by weight, whereas higher UFR was more strongly associated with mortality outcomes in higher quintiles of body size. These results were consistent to those of prior studies [27]. Obese or higher weight patients may have greater underlying $\mathrm{CV}$ risk and thereby may be more vulnerable to complications associated with higher UFRs (i.e., intradialytic hypotension, myocardial stunning). Further studies are needed to understand the underlying pathophysiology contributing to the more potent effect of UFR on mortality in higher weight patients.

There are several limitations that need to be mentioned. Although we adjusted for demographics, comorbidities, and laboratory covariates, we cannot exclude the possibility of residual confounding due to the observational nature of this study. In addition, we cannot exclude the possibility of residual confounding as data on baseline residual renal function or volume status at the time of dialysis initiation were highly missing in the cohort, and thus we cannot adequately examine the relationship of UFR and mortality through adjusting for these factors. Despite these limitations, the strengths of our study includes the examination of a large, nationally representative cohort of 110,880 incident HD patients with uniformly measured laboratory data from a single laboratory facility and a long follow-up for up to 5 years.

This observational study showed that higher UFR is independently associated with higher all-cause and CV mortalities in incident HD patients. Also, there were incremental and almost linear associations between UFR and mortality irrespective of gender, race, age, urine volume, dialysis treatment time, ultrafiltration, and post-dialysis systolic BP categories. Further studies examining the effects of UFR at HD initiation are warranted.

\section{Acknowledgments and Disclosure Statement}

K.K.-Z. has received honoraria from Genzyme/Sanofi and Shire and was the medical director of DaVita Harbor-UCLA/MFI in Long Beach, CA, during 2007-2012. Other authors have not declared any conflict of interest. We thank DaVita Clinical Research $^{\circledR}$ for providing the clinical data for this research. 


\section{Support and Financial Disclosure}

The work is supported by K.K.-Z. NIH (NIDDK) grants K24DK091419, R01-DK078106, R01-DK95668, a philanthropic grant from Mr. Harold Simmons, Mr. Louis Chang, and Aveo, Inc.

\section{Statement of Ethics}

The study was approved by the Institutional Review Board University of California, Irvine. The requirement for a written consent was exempt due to the large sample size, patient anonymity, and non-intrusive nature of the research.

\section{Author Contributions}

T.W.K., C.P.K., E.S., and K.K.Z. contributed to the study concept and design. K.K.Z. acquired the data. T.W.K., M.S., and E.S. conducted statistical analyses and interpretation with advice from T.I.C., T.H.K., J.C., C.P.K., and K.K.Z.; T.W.K., M.S., and E.S. drafted the manuscript; and T.I.C., T.H.K., J.C., V.R., C.P.K., and K.K.Z. critically revised it for important intellectual content. All authors contributed to the preparation of the report and approved the final version. We have included a conflict of interest disclosure statement in the manuscript.

\section{References}

1 US Renal Data System: USRDS 2008 Annual Data Report: Atlas of End-Stage Renal Disease in the United States. Bethesda, National Institutes of Health, National Instututes of Diabetes and Digestive and Kidney Disease, 2008.

$>2$ Weiner DE, Tighiouart H, Amin MG, Stark PC, MacLeod B, Griffith JL, Salem DN, Levey AS, Sarnak MJ: Chronic kidney disease as a risk factor for cardiovascular disease and allcause mortality: a pooled analysis of community-based studies. J Am Soc Nephrol 2004; 15:1307-1315.

-3 Rocco MV, Yan G, Heyka RJ, Benz R, Cheung AK: Risk factors for hypertension in chronic hemodialysis patients: baseline data from the HEMO study. Am J Nephrol 2001;21:280-288.

$\checkmark 4$ Testa A, Beaud JM: The other side of the coin: interdialytic weight gain as an index of good nutrition. Am J Kidney Dis 1998;31:830-834.

5 Ifudu O, Uribarri J, Rajwani I, Vlacich V, Reydel K, Delosreyes G, Friedman EA: Relation between interdialytic weight gain, body weight and nutrition in hemodialysis patients. Am J Nephrol 2002;22:363-368.

6 Sarkar SR, Kotanko P, Levin NW: Interdialytic weight gain: implications in hemodialysis patients. Semin Dial 2006;19:429-433.

7 Sharpe N: Left ventricular remodeling: pathophysiology and treatment. Heart Fail Monit 2003;4:55-61.

$>8$ Held PJ, Levin NW, Bovbjerg RR, Pauly MV, Diamond LH: Mortality and duration of hemodialysis treatment. JAMA 1991;265:871875.

9 Valderrábano F: Weekly duration of dialysis treatment - does it matter for survival? Nephrol Dial Transplant 1996;11:569-572.

-10 Shinzato T, Nakai S, Akiba T, Yamazaki C, Sasaki R, Kitaoka T, Kubo K, Shinoda T, Kurokawa K, Marumo F, Sato T, Maeda K: Survival in long-term haemodialysis patients: results from the annual survey of the Japanese Society for Dialysis Therapy. Nephrol Dial Transplant 1997; 12:884-888.

$\checkmark 11$ Innes A, Charra B, Burden RP, Morgan AG, Laurent G: The effect of long, slow hemodi- alysis on patient survival. Nephrol Dial Transplant 1999;14:919-922.

-12 Saran R, Bragg-Gresham JL, Levin NW, Twardowski ZJ, Wizemann V, Saito A, Kimata N, Gillespie BW, Combe C, Bommer J, Akiba T, Mapes DL, Young EW, Port FK: Longer treatment time and slower ultrafiltration in hemodialysis: associations with reduced mortality in the DOPPS. Kidney Int 2006;69:1222-1228.

13 Movilli E, Gaggia P, Zubani R, Camerini C, Vizzardi V, Parrinello G, Savoldi S, Fischer MS, Londrino F, Cancarini G: Association between high ultrafiltration rates and mortality in uraemic patients on regular haemodialysis. A 5-year prospective observational multicentre study. Nephrol Dial Transplant 2007;22:3547-3552.

14 Flythe JE, Kimmel SE, Brunelli SM: Rapid fluid removal during dialysis is associated with cardiovascular morbidity and mortality. Kidney Int 2011;79:250-257.

15 Kuttykrishnan S, Kalantar-Zadeh K, Arah OA, Cheung AK, Brunelli S, Heagerty PJ, Katz R, Molnar MZ, Nissenson A, Ravel V, Streja E, Himmelfarb J, Mehrotra R: Predictors of treatment with dialysis modalities in observational studies for comparative effectiveness research. Nephrol Dial Transplant 2015;30:1208-1217.

16 Lopez-Gomez JM, Villaverde M, Jofre R, Rodriguez-Benitez P, Perez-Garcia R: Interdialytic weight gain as a marker of blood pressure, nutrition, and survival in hemodialysis patients. Kidney Int Suppl 2005;67:S63-S68.

-17 Movilli E, Camerini C, Gaggia P, Zubani R, Feller P, Poiatti P, Pola A, Carli O, Cancarini $\mathrm{G}$ : Magnitude of end-dialysis overweight is associated with all-cause and cardiovascular mortality: a 3-year prospective study. Am J Nephrol 2013;37:370-377.

18 Lee MJ, Doh FM, Kim CH, Koo HM, Oh HJ, Park JT, Han SH, Yoo TH, Kim YL, Kim YS, Yang CW, Kim NH, Kang SW: Interdialytic weight gain and cardiovascular outcome in incident hemodialysis patients. Am J Nephrol 2014;39:427-435.

19 Saran R, Bragg-Gresham JL, Rayner HC, Goodkin DA, Keen ML, Van Dijk PC, Ku- rokawa K, Piera L, Saito A, Fukuhara S, Young EW, Held PJ, Port FK: Nonadherence in hemodialysis: associations with mortality, hospitalization, and practice patterns in the DOPPS. Kidney Int 2003;64: 254-262.

20 Kalantar-Zadeh K, Regidor DL, Kovesdy CP, Van Wyck D, Bunnapradist S, Horwich TB, Fonarow GC: Fluid retention is associated with cardiovascular mortality in patients undergoing long-term Hemodialysis. Circulation 2009;119:671-679.

21 Tisler A, Akocsi K, Borbas B, Fazakas L, Ferenczi S, Gorogh S, Kulcsar I, Nagy L, Samik J, szegedi J, Toth E, Wagner G, Kiss I: The effect of frequent or occasional dialysis-associated hypotension on survival of patients on maintenance haemodialysis. Nephrol Dial Transplant 2003;18:2601-2605.

22 Burton JO, Jefferies HJ, Selby NM, McIntyre CW: Hemodialysis-induced cardiac injury: determinants and associated outcomes. Clin J Am Soc Nephrol 2009;4:914-920.

23 Flythe JE, Xue H, Lynch KE, Curhan GC, Brunelli SM: Association of mortality risk with various definitions of intradialytic hypotension. J Am Soc Nephrol 2015;26:724-734.

24 Miller JE, Kovesdy CP, Nissenson AR, Mehrotra R, Streja E, Van Wyck D, Greenland S, Kalantar-Zadeh K: Association of hemodialysis treatment time and dose with mortality and the role of race and sex. Am J Kidney Dis 2010;55:100-112.

25 Flythe JE, Curhan GC, Brunelli SM: Disentangling the ultrafiltration rate-mortality association: the respective roles of session length and weight gain. Clin J Am Nephrol 2013;8: 1151-1161.

26 Twardowski ZJ: Treatment time and ultrafiltration rate are more important in dialysis prescription than small molecule clearance. Blood Purif 2007;25:90-98.

27 Assimon MM, Wenger JB, Wang L, Flythe JE: Ultrafiltration rate and mortality in maintenance hemodialysis patients. Am J Kidney Dis 2016;68:911-922.
10 Nephron DOI: $10.1159 / 000486323$
Kim/Chang/Kim/Chou/Soohoo/Ravel/ Kovesdy/Kalantar-Zadeh/Streja 